\title{
MRI-based assessment of liver perfusion and hepatocyte injury in the murine model of acute hepatitis
}

\author{
Katarzyna Byk ${ }^{1} \cdot$ Krzysztof Jasinski $^{1} \cdot$ Zaneta Bartel $^{1} \cdot$ Agnieszka Jasztal $^{2} \cdot$ \\ Barbara Sitek $^{2} \cdot$ Boguslaw Tomanek $^{1,3} \cdot$ Stefan Chlopicki $^{2,4} \cdot$ Tomasz Skorka $^{1}$
}

Received: 14 February 2016 / Revised: 9 April 2016 / Accepted: 20 April 2016 / Published online: 9 May 2016

(C) The Author(s) 2016. This article is published with open access at Springerlink.com

\begin{abstract}
Objective To assess alterations in perfusion and liver function in the concanavalin A (ConA)-induced mouse model of acute liver failure (ALF) using two magnetic resonance imaging (MRI)-based methods: dynamic contrast-enhanced MRI (DCE-MRI) with Gd-EOB-DTPA contrast agent and arterial spin labelling (ASL).

Materials and methods $\mathrm{BALB} / \mathrm{c}$ mice were studied using a 9.4 T MRI system. The IntraGateFLASH ${ }^{\mathrm{TM}}$ and FAIR-EPI pulse sequences were used for optimum mouse abdomen imaging.

Results The average perfusion values for the liver of the control and ConA group were equal to $245 \pm 20$ and $200 \pm 32 \mathrm{ml} / \mathrm{min} / 100 \mathrm{~g}$ ( $p=0.008$, respectively). DCE-MRI showed that the time to the peak of the image enhancement was $6.14 \pm 1.07 \mathrm{~min}$ and $9.72 \pm 1.69 \mathrm{~min}$ in the control and ConA group ( $p<0.001$, respectively), while the rate of the contrast wash-out in the control and ConA group was $0.037 \pm 0.008$ and $0.021 \pm 0.008 \mathrm{~min}^{-1}$ ( $p=0.004$, respectively). These results were consistent with hepatocyte injury in the ConA-treated mice as confirmed by histopathological staining.
\end{abstract}

Tomasz Skorka

Tomasz.Skorka@ifj.edu.pl

1 Department of Magnetic Resonance Imaging, Institute of Nuclear Physics, Polish Academy of Sciences, Krakow, Poland

2 Jagiellonian Centre for Experimental Therapeutics (JCET), Jagiellonian University, Krakow, Poland

3 Division of Medical Physics, Department of Oncology, University of Alberta, Edmonton, Alberta, Canada

4 Department of Experimental Pharmacology, Chair of Pharmacology, Medical College, Jagiellonian University, Krakow, Poland
Conclusions Both the ASL and DCE-MRI techniques represent a reliable methodology to assess alterations in liver perfusion and hepatocyte integrity in murine hepatitis.

Keywords Acute liver failure · Arterial spin labelling ·

DCE-MRI · Empirical mathematical modelling · Perfusion

\section{Introduction}

Acute liver failure is a disease associated with high mortality and multiorgan dysfunction [1]. The aetiology of ALF is complex [2] as it can be caused by viruses, reactions to drugs, vascular diseases or metabolic syndromes $[2,3]$. ALF is associated with a primary liver dysfunction, activation of pro-inflammatory responses including proinflammatory cytokines, acute phase proteins [4], and activation of liver sinusoidal endothelial cells (LSEC), Kupffer cells and recruitment of immune cells [5]. ALF invariably leads to the loss of hepatocyte integrity as mirrored by the elevated levels of the liver specific enzymes [alanine aminotransferase (ALT) and aspartate aminotransferase (AST)] in blood [6]. It also influences the functional state of the liver leading to haemodynamic disturbances, inappropriate vasodilatation and reduction of perfusion [7-9].

The ConA-induced model of ALF was originally used for studies of the pathophysiology of lymphocyte T-dependent liver injury and in pre-clinical studies on liver inflammation [10]. ConA-induced hepatitis involves cooperative activation of natural killer T (NKT) with conventional T cells and Kupffer cells and IL-4-, TNF- $\alpha$ - and IFN- $\gamma$-mediated liver inflammation in mice with subsequent development of hepatocellular apoptosis and necrosis [10-12] that eventually results in endothelium disintegration and necrosis of hepatocytes [10]. 
MRI-based methods can be applied for the quantitative measurement of liver function in hepatitis using small animals models; however the studies are challenging because of the low signal-to-noise ratio and respiratory artefacts. Animals with advanced ALF may develop complications such as acute lung injury or respiratory distress syndrome [1] causing uncontrolled motion and thus further image distortions. Therefore proper pulse sequences must be selected to ensure optimum image quality.

Complex diagnosis of ALF includes investigation of hepatocyte integrity and liver perfusion. The MRI technique, arterial spin labelling (ASL), was developed for perfusion measurements [13]. The double-triggered flowsensitive alternating inversion recovery (FAIR) [14] with rapid single-shot echo planar imaging (EPI) acquisition is a particular type of ASL. FAIR registers blood inflows from different directions [14]; therefore it is advantageous over other types of ASL modalities for perfusion imaging of the liver with a complex microcirculatory bed. Moreover, the FAIR-EPI technique allows obtaining maps of the $T_{1}$ relaxation time, which was previously reported as an indicator of liver disease $[15,16]$. DCE-MRI was used in studies of liver injury $[17,18]$, but was also reported to be useful for assessment of perfusion changes [19, 20]. The $T_{1}$-weighted fast gradient echo sequences are used for DCE-MRI, but they need triggering. Therefore the selfgated IntraGateFLASH ${ }^{\mathrm{TM}}$ [21] technique seems valuable especially for small animal imaging. This sequence allows continuous scanning in steady-state conditions and retrospective removal of the data altered by respiration artifacts. IntraGateFLASH ${ }^{\mathrm{TM}}$ has been used in studies of mouse heart mapping [22] and mouse cholangiography [23], for example.

The aim of our work was to measure perfusion and hepatocyte injury in the mouse model of acute hepatitis induced with ConA with the use of two complementary MRI-based methods. Alterations in the hepatocyte integrity and functional state of microcirculation of the liver were assessed as they are important factors determining liver function and response to injury.

\section{Materials and methods}

\section{Animals studies}

Fourteen 3-month-old BALB/c mice were used in this study. Acute hepatitis was induced by ConA (SigmaAldrich, USA) administered intravenously. MRI measurements were performed $24 \mathrm{~h}$ after ConA injection. The animals were divided into the control and ConA group ( $n=7$ in each group). The treated group received $8 \mathrm{mg}$ / kg b.w. ConA in $1 \mathrm{M}$ phosphate-buffered saline (PBS), and the control group received the same volume of saline. The mice were fed a standard laboratory diet and were maintained in a 12-h light/dark cycle at $23{ }^{\circ} \mathrm{C}$ temperature. All procedures were approved by the local Ethics Committee on Animal Testing.

\section{MRI}

The MRI experiments were performed using a $9.4 \mathrm{~T} / 21-\mathrm{cm}$ (BioSpec 94/20 USR, Bruker, Germany) horizontal scanner equipped with a high-performance gradient system and a 36-mm-diameter ${ }^{1} \mathrm{H}$ quadrature transmit/receive radio-frequency (RF) volume coil.

The animals were anesthetised with $2 \%$ isoflurane in a mixture of air and oxygen delivered via a nose cone. The anesthetic was reduced to $1.7 \%$ when animals were placed in the magnet. Electrocardiography (ECG), respiration and temperature were monitored with the Monitoring and Gating System (SA Instruments Inc., Stony Brook, NY, USA). Body temperature was maintained at $37^{\circ} \mathrm{C}$. The animals were placed prone and maintained throughout the experiments, usually $2.5 \mathrm{~h}$ long. Before the MRI experiments $375 \mu \mathrm{l}$ of saline was injected intraperitoneally to prevent dehydration. A catheter was inserted into the tail vein for contrast agent administration.

The imaging slice was positioned perpendicularly to the large blood vessels (aorta, vena cava and postal vein).

ASL imaging was conducted using the FAIR-EPI pulse sequence (Fig. 1) with the following parameters: $\mathrm{TE}=6.324 \mathrm{~ms}, \mathrm{TR}=8000 \mathrm{~ms}, \mathrm{NA}=4$, $\mathrm{FOV}=45 \times 30 \mathrm{~mm}$, matrix size $=96 \times 64$, slice thickness $=1 \mathrm{~mm}$, selective inversion slice thickness $=2.5 \mathrm{~mm}$ and 10 inversion recovery times (IRs) from 50 to $4500 \mathrm{~ms}$. Fat suppression was used with a frequency selective $90^{\circ}$ pulse. The total measurement time was approximately $10 \mathrm{~min}$.

DCE-MRI was performed using the IntraGateFLASH ${ }^{\mathrm{TM}}$ sequence with the following parameters: $\mathrm{TE}=0.838 \mathrm{~ms}$, $\mathrm{TR}=4.212 \mathrm{~ms}, \mathrm{NA}=1500$ or 200 , flip angle $=18^{\circ}$, $\mathrm{FOV}=30 \times 30 \mathrm{~mm}$, matrix size $=128 \times 128$, slice thickness $=1 \mathrm{~mm}$ and navigator slice thickness $=8 \mathrm{~mm}$. The total measurement time was $90 \mathrm{~min} ; 10 \mu \mathrm{l} / 1 \mathrm{~g} \mathrm{~b} . w$. of $0.0025 \mathrm{M}$ hepatotropic gadolinium-based contrast agent (Primovist $^{\circledR}$, Bayer Schering Pharma AG, Germany) [24] was injected into the tail vein 2 min after the first image acquisition. MR images were collected in three series (150 images each), each $10 \mathrm{~min}$ long, a total of $30 \mathrm{~min}$, to observe the contrast uptake and the maximum enhancement of liver tissue with high temporal resolution; 1500 averages were used for each series. During the remaining $1 \mathrm{~h}$, one image was acquired every $10 \mathrm{~min}$ with 200 averages. 


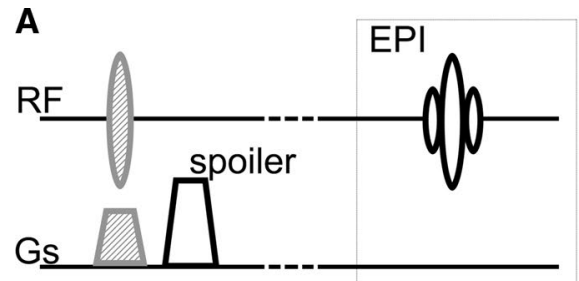

B

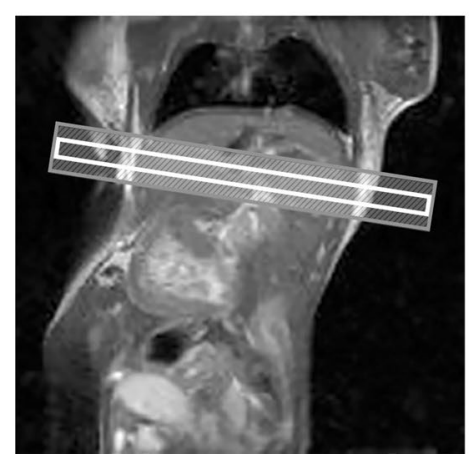

Fig. 1 The FAIR pulse sequence with EPI readout. Two MR images are necessary for perfusion imaging, one with selective $(\mathbf{a}, \mathbf{b})$ and one with non-selective inversion of spins (c, d). a The RF pulse is applied simultaneously with the slice selective gradient (shaded grey) for the selective inversion of spins. The slice gradient is followed by the spoiler gradient for magnetisation refocussing. b The selective

Immediately after the MR experiments the animals were killed, blood was collected for analysis, and the livers were harvested for histological staining.

\section{Data analysis}

Perfusion and $T_{1}$ maps were created using ASL Perfusion Macro software (ParaVision 5.1, Bruker, Germany). The $T_{1}$ values were obtained from the control $T_{1}$ maps with nonselective spin inversion. The threshold was applied to the perfusion maps to reject false values (higher than $500 \mathrm{ml} /$ $\mathrm{min} / 100 \mathrm{~g}$ or negative). ImageJ software (NIH, USA) was used for the thresholding. Three regions of interest (ROI) placed in the liver parenchyma were used for analysis.

The time courses of the DCE-MRI images were processed with ImageJ. An oval-shaped ROI comprising 137 pixels for each MR image was placed in the right liver lobe. The mean values $( \pm \mathrm{SD})$ of the signal intensities within ROIs were calculated for each image. The data were processed with Origin Pro 8.6.0 (OriginLab, MA, USA). The signal-time curves were normalised by averaging the signal values of the pre-contrast images and subtracting the average value from the entire time course. The final curves were fitted with the empirical mathematical model (EMM) proposed by Fan et al. [25]:

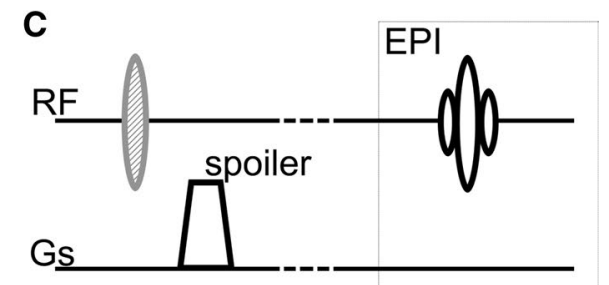

D

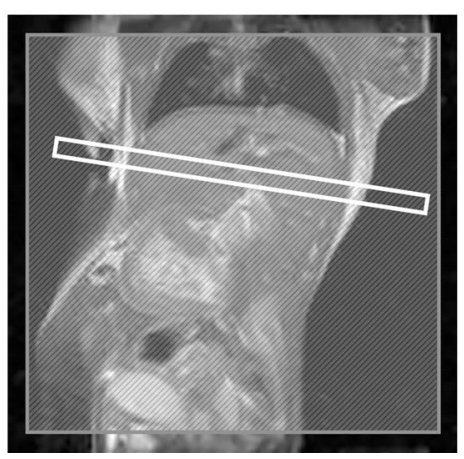

inversion is wider than the selected slice (in white) to ensure uniform inversion. c As a control image, the RF pulse is applied without the slice-selective gradient providing excitation of all spins. d The area of non-selective inversion covers the entire sensitive region of the RF coil. The FAIR pulse sequence is followed by the EPI sequence to create the MR image [14]

$C(t)=A \cdot\left(1-e^{-\alpha t}\right)^{q} \cdot e^{-\beta t} \cdot \frac{1+e^{-\gamma t}}{2}$,

where $A$ is the upper limit of the signal, $\alpha$ is the rate of the contrast uptake $(1 / \mathrm{min}), \beta$ is the rate of the contrast washout ( $1 / \mathrm{min}), \gamma$ is the initial rate of the contrast wash-out (1/ $\min$ ), and $q$ is the parameter related to the slope of the early uptake and the curvature of the transition from uptake to wash-out. Four additional parameters were also calculated: time to peak $\left[T_{\text {peak }},(\mathrm{min})\right]$, enhancement slope [ES, (1/ min)], area under the curve (AUC) and elimination halflife time $\left[T_{1 / 2},(\min )\right]$. $T_{\text {peak }}$ was calculated according to the formula proposed by Fan et al. [25]. ES was calculated as the ratio of $C_{\text {max }} / T_{\text {peak }}$, where $C_{\max }$ was determined directly from the signal-time graph. AUC was computed from time 0-90 $\mathrm{min}$. The $T_{1 / 2}$ was assessed by fitting the exponential curve to the wash-out slope.

\section{Biochemical and histopathological analysis}

Following the MR measurements blood samples were obtained from the renal vein on ethylenediaminetetraacetic acid (EDTA) and centrifuged for $10 \mathrm{~min}$ at $1000 \mathrm{~g}$ at a temperature of $4{ }^{\circ} \mathrm{C}$ (Sigma 2-16PK, Sigma, Germany). Blood plasma was frozen at $-80{ }^{\circ} \mathrm{C}$ and stored. The content of 
ALT and AST in blood plasma was determined with calorimetric methods using an ABX Penta 400 biochemical analyzer (Horiba Medical, Japan).

Following the blood sampling, the livers were harvested and fixed in $4 \%$ formaldehyde phosphate buffer solution. The liver lobes were embedded in paraffin. The sections were $5 \mu \mathrm{m}$ thick and were cut in two areas (two slices per area) approximately $100 \mu \mathrm{m}$ apart. The regions with the necrotic (haemorrhagic and coagulative necrosis) and the collective tissue changes were evaluated as a percentage of the total section area. The infiltration of vessel walls by immune cells was classified on a fourpoint scale, where the score 0 was assigned to the tissue without infiltration of vessel walls and the score 3 was assigned to the samples in which the infiltration was noticed in each vessel. The samples were stained with haematoxylin and eosin (H\&E) and Gomori trichrome (TRICHROME).

\section{Statistical analysis}

All parametric parameters were presented as (mean \pm STD); histological parameters were presented as median. The data, except for the histological information, was verified for normality with the Shapiro-Wilk test $(p=0.05)$. Parameters with normal distribution were analysed with the $t$ test $(p=0.05)$. The Mann-Whitney $U$ test was applied $(p=0.05)$ to qualitative parameters determined on the interval scale and parametric parameters with substantial distribution. Correlations among perfusion, EMM parameters and histological outcomes were tested with the Spearman rank order correlation $(p=0.05)$. Statistical analysis was performed with Statistica 8.0 (Stat Soft, Tulsa, OK, USA).

\section{Results}

\section{Development of acute liver injury after ConA injection}

\section{Weight of the animals}

The weight of the animals was measured just before and $24 \mathrm{~h}$ after ConA/saline injection. The weight of the ConAtreated group was $20.9 \pm 0.8 \mathrm{~g}$ and $19.9 \pm 1.1 \mathrm{~g}$ before and $24 \mathrm{~h}$ after ConA, respectively. The weight of the control animals remained stable at $20.1 \pm 1.4 \mathrm{~g}$ before and $24 \mathrm{~h}$ after the saline injection. In both groups, the change in the weight was not statistically significant $(p>0.05)$.

\section{Histopathology of the liver}

Histological staining of the liver tissue from the control group showed apoptosis of single hepatocytes and traces of inflammation (Fig. 2a). No other abnormalities were observed. Histological cross-sections of the control animals were normal. In the ConA-treated group pathological changes such as hyperaemia, coagulative and haemorrhagic necrosis, inflammatory infiltration in the blood vessels wall and liver parenchyma, embolism in the vessel lumens, disintegration of the endothelium, hepatocyte blebs and haemorrhage were detected (Fig. 2b, c; Table 1). Numerous foci of necrosis scattered throughout the parenchyma in the livers of the ConA group were observed. Cellular infiltration of the parenchyma was noticed around and in the areas of necrosis (Fig. 2b).

\section{Biochemical parameters}

Biochemical analysis showed substantially elevated levels of enzymes in blood plasma in the ConA group. ALT
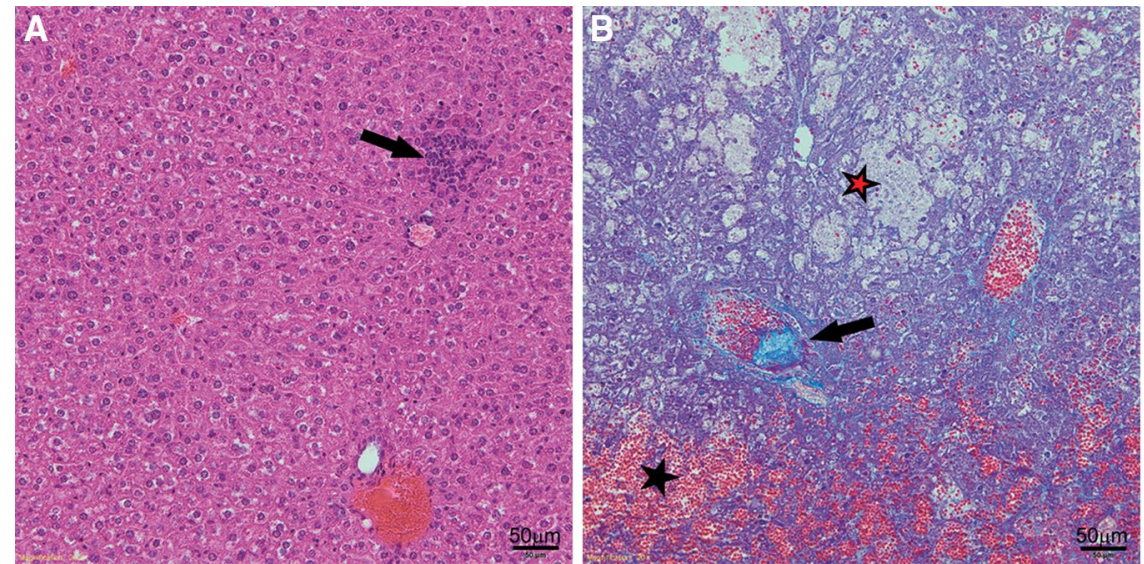

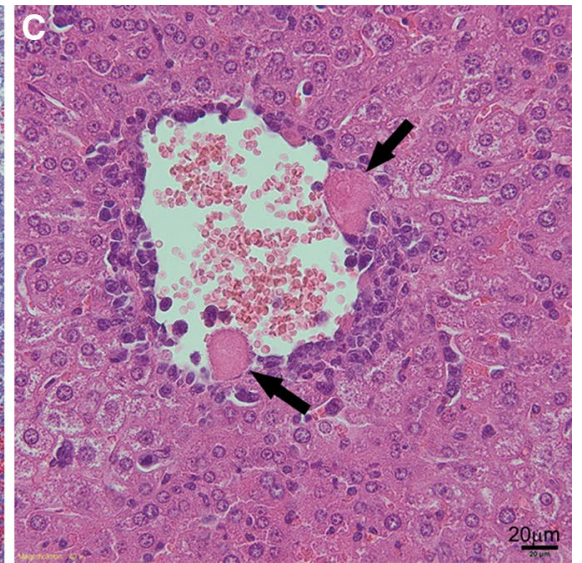

Fig. 2 Representative microphotographs of cross sections of the livers harvested from a the control group (H\&E staining; $\times 200) ; \mathbf{b}$ ConA group (TRICHROME staining; $\times 200)$; c ConA group $(\mathrm{H} \& \mathrm{E}$ staining; $\times 400)$. A small inflammation focus is visible (arrow) in a. Haemorrhage (marked with a black star), haemorrhagic necrosis (marked with a red star) and embolism in a vessel (arrow) are visible in b. Hepatocyte blebs (arrows) are visible in c 
Table 1 Histopathological evaluation of control mice and mice with liver injury induced by ConA

\begin{tabular}{lllllll}
\hline Parameter & \multicolumn{2}{l}{$\begin{array}{l}\text { Control group } \\
n=7\end{array}$} & \multicolumn{2}{l}{$\begin{array}{l}\text { ConA group } \\
n=7\end{array}$} & $p$ value \\
\cline { 2 - 3 } & Median & Rank sum & Median & Rank sum n & \\
\hline $\begin{array}{c}\text { Haemorrhagic } \\
\text { necrosis [\%] }\end{array}$ & 0 & 42 & 0 & 63 & 0.2 \\
$\begin{array}{c}\text { Coagulative } \\
\text { necrosis [\%] }\end{array}$ & 0 & 31.5 & 10 & 73.5 & 0.009 \\
$\begin{array}{c}\text { Vessel wall infil- } \\
\text { tration [a.u.] }\end{array}$ & 0 & 28 & 2 & 77 & 0.002 \\
$\begin{array}{c}\text { Tissue changes } \\
{[\%]}\end{array}$ & 0 & 28 & 40 & 77 & 0.002 \\
\hline
\end{tabular}

Hyperaemia, coagulative necrosis and vessel wall infiltration were observed in the samples from the ConA group. A median and rank sum from the Mann-Whitney $U$ test are shown. Test results were assumed to be significantly different at $p<0.05$ (italics)

was equal to $38 \pm 7$ and $1627 \pm 1075 \mathrm{U} / \mathrm{l}$ in the control and ConA group, respectively, while the corresponding AST values were $61 \pm 15$ and $1977 \pm 1285$ U/l. The Mann-Whitney $U$ test showed that these parameters were significantly different between the groups with the following $p$ values: ALT $(p<0.001)$; AST $(p<0.001)$.

\section{Assessment of perfusion and $T_{1}$ relaxation time with arterial spin labelling}

The examples of the perfusion maps are presented in Fig. 3a, b. Perfusion in the liver ROIs was equal to $245 \pm 20 \mathrm{ml} / \mathrm{min} / 100 \mathrm{~g}$ in the healthy group and $200 \pm 32 \mathrm{ml} / \mathrm{min} / 100 \mathrm{~g}$ in the ConA group (Fig. 3c). The $t$ test indicated that the perfusion values were significantly different $(p=0.008)$ between the control and ConA group and decreased by $18 \%$ in the treated group. A threshold was applied to the pixels with perfusion values higher than $500 \mathrm{ml} / \mathrm{min} / 100 \mathrm{~g}$ of tissue. As a result, the pixels in the central area of the initial MR images were removed because of the presence of thevena cava and portal vein.

The examples of the $T_{1}$ maps are presented in Fig. $3 d$ and e. The $T_{1}$ relaxation time in the livers of the control group across all animals was equal to $1249 \pm 91 \mathrm{~ms}$ while in the ConA group it was $1597 \pm 130 \mathrm{~ms}$ (Fig. 3f). The difference in $T_{1}$ between the control and treated group was significant $(p<0.001)$.
A

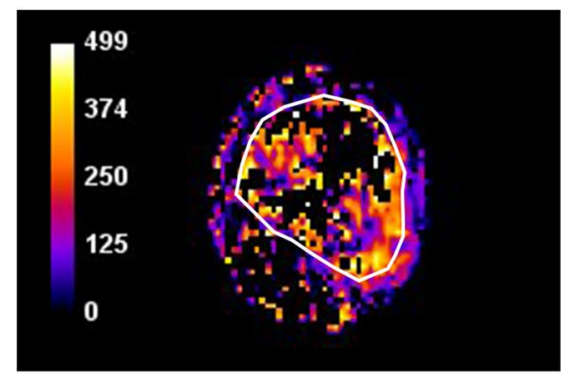

B
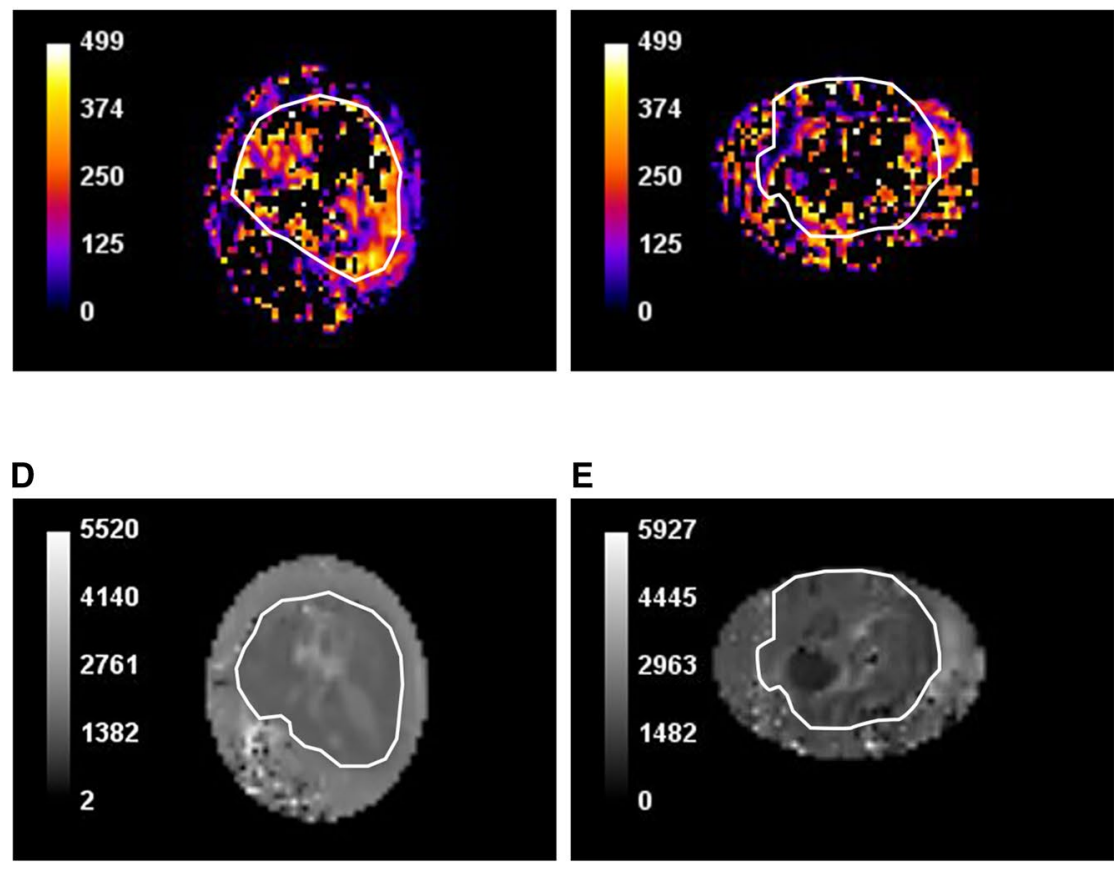

E

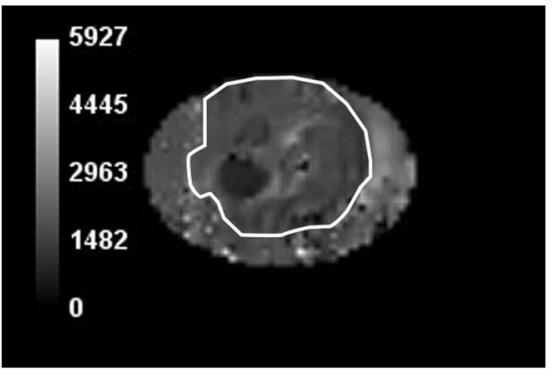

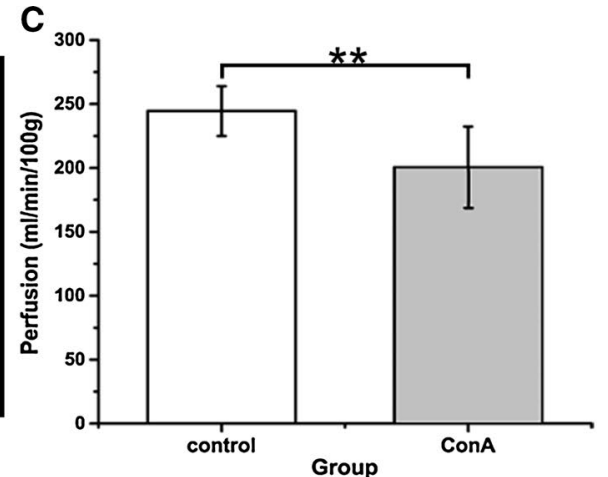

$\mathbf{F}$

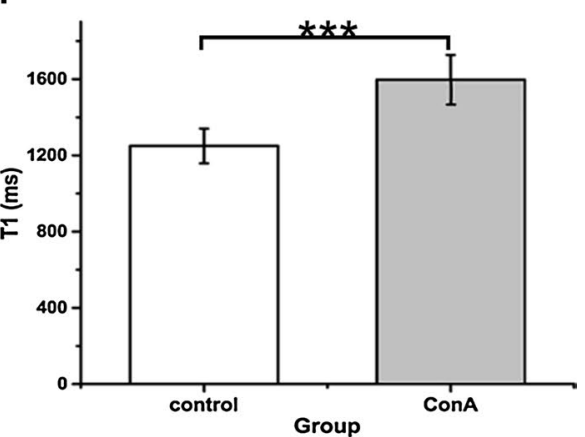

Fig. 3 Examples of the perfusion $[\mathrm{ml} / \mathrm{min} / 100 \mathrm{~g}](\mathbf{a}, \mathbf{b})$ and $T_{1}$ [ms] (c, e) maps. Both maps were rescaled from the original size to $256 \times 171$ pixels. Thresholding of pixel values greater than $500 \mathrm{ml} /$ $\mathrm{min} / 100 \mathrm{~g}$ was applied to the perfusion maps. Perfusion (c) and $T_{1}$ values (f) were assessed for seven control and seven treated animals. a Perfusion map of the control liver. b Perfusion map of the
ALF liver. c Perfusion values within ROIs in the livers of the control and ConA group. The difference in perfusion between the control and ConA group is statistically significant $\left({ }^{* *} p=0.008\right)$. $\mathbf{d} T_{1}$ map of control liver. e $T_{1}$ map of ALF liver. $\mathbf{f} T_{1}$ values in the control and ConA group are significantly different $(* * * p<0.001)$. Areas of liver in MR images (a-d) are contoured in white 
Fig. 4 Examples of the time courses of signal changes in the control and ConA group with the fitted empirical mathematical model. The differences in the curvatures of the enhancement peaks and wash-out regions of the curves for the ConA and the control are visible. The corresponding DCEMR images of the liver from the control group are shown beneath the horizontal axis: (1) pre-contrast; (2) 6 min after contrast injection (maximum enhancement of liver parenchyma is visible, $T_{\text {peak }}$ in control group was equal to $6.14 \mathrm{~min}$ ); (3) $30 \mathrm{~min}$; (4) $60 \mathrm{~min}$; (5) 90 min after contrast injection (contrast is washed out from the liver tissue). During the initial $30 \mathrm{~min}$, MR images were acquired in three series, 150 images each. After $30 \mathrm{~min}$, one image was acquired every $10 \mathrm{~min}$

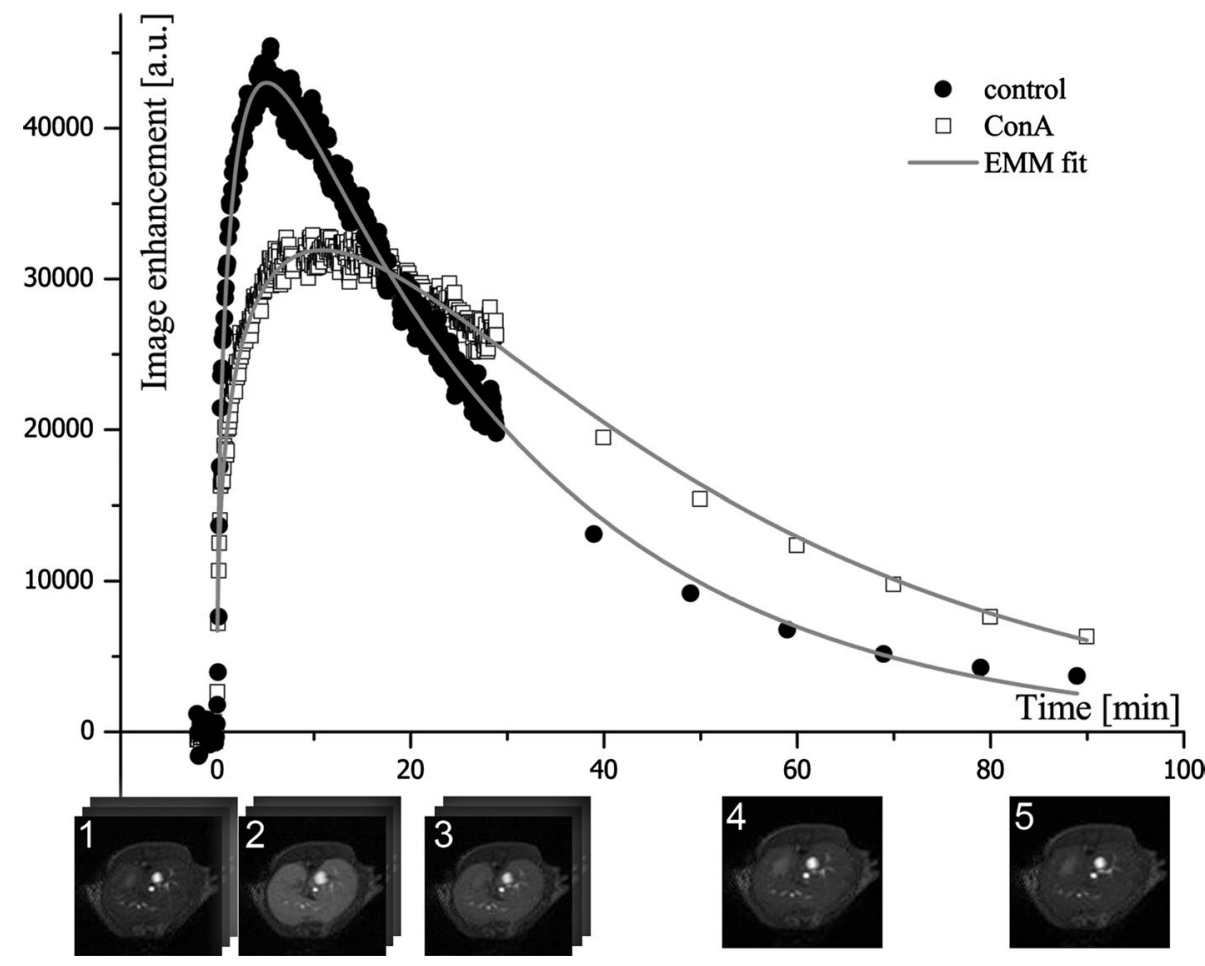

\section{Assessment of the hepatocyte integrity with the DCE-MRI and empirical mathematical model}

The Gd-EOB-DTPA compound is a hepatocyte-specific contrast agent that is taken up by living hepatocytes. As shown in Fig. 4 the Gd-EOB-DTPA signal displays different patterns in the control and ConA mice. Gd-EOB-DTPA was washed away faster in the livers of the control animals than in the livers of the ConA animals. The MR signal intensity of parenchyma in the controls returned to the preinjection value within $90 \mathrm{~min}$. In the ConA group the intensity of the liver MR images remained elevated until the end of the measurements (Fig. 4).

To further quantify differences in the Gd-EOB-DTPA wash-out between the control and ConA group, the EMM equation was fitted to the experimental data $\left(R^{2}>0.98\right)$. The values of the parameters calculated from the EMM and $p$ values are shown in Table 2. The rate of the initial wash out $(\gamma)$ shows substantial distribution and was tested with the Mann-Whitney $U$ test. The remaining parameters were tested with the $t$ test. Five parameters were statistically different between the control and the ConA group: $\beta(p=0.004), T_{\text {peak }}(p<0.001)$, ES $(p=0.006), T_{1 / 2}$ $(p<0.001)$ and AUC $(p=0.003)$. The parameters $\beta$ and $T_{1 / 2}$ describe the wash-out slope of the DCE-MRI curve. The significant difference in these parameters between the groups confirmed that transport of the contrast agent via hepatocytes was disturbed by the ALF processes. The value of $\beta$ was equal to $0.037 \pm 0.008$ and $0.021 \pm 0.008$
Table 2 Parameters obtained by fitting the EMM equation to the experimental data for both groups

\begin{tabular}{|c|c|c|c|}
\hline $\begin{array}{l}\text { Param- } \\
\text { eter }\end{array}$ & $\begin{array}{l}\text { Control group } \\
n=7\end{array}$ & $\begin{array}{l}\text { ConA group } \\
n=7\end{array}$ & $p$ value \\
\hline$A$ & $57,100 \pm 9669$ & $67,355 \pm 31747$ & 0.43 \\
\hline $\begin{array}{l}\alpha[1 / \\
\quad \min ]\end{array}$ & $0.20 \pm 0.11$ & $0.11 \pm 0.08$ & 0.1 \\
\hline$q$ & $0.432 \pm 0.064$ & $0.380 \pm 0.064$ & 0.15 \\
\hline $\begin{array}{l}\beta[1 / \\
\min ]\end{array}$ & $0.037 \pm 0.008$ & $0.021 \pm 0.008$ & 0.004 \\
\hline $\begin{array}{l}\gamma[1 / \\
\min ]\end{array}$ & $0.001 \pm 0.002$ (r.s.43) & $0.004 \pm 0.007$ (r.s.62) & 0.25 \\
\hline $\begin{array}{l}T_{\text {peak }} \\
\text { [min] }\end{array}$ & $6.14 \pm 1.07$ & $9.72 \pm 1.69$ & $<0.001$ \\
\hline $\begin{array}{r}\mathrm{ES}[1 / \\
\mathrm{min}]\end{array}$ & $6587 \pm 1731$ & $4002 \pm 1113$ & 0.006 \\
\hline $\begin{array}{l}T_{1 / 2} \\
{[\mathrm{~min}]}\end{array}$ & $31.7 \pm 7.3$ & $66.5 \pm 10.5$ & $<0.001$ \\
\hline AUC & $1,312,380 \pm 252,093$ & $1,786,030 \pm 229,748$ & 0.003 \\
\hline
\end{tabular}

The parameters, except $\gamma$, were tested with the $t$ test. $\gamma$ was tested with the Mann-Whitney $U$ test; rank sum is placed in parentheses. Statistical significance was assumed at $p<0.05$ (italics)

$1 / \mathrm{min}$ in the control and ConA group, respectively, while the corresponding $T_{1 / 2}$ values were $31.7 \pm 7.3$ and $66.5 \pm 10.5 \mathrm{~min}$. A significant difference was also observed in the AUC parameter, which was larger in the ConA group than in the control group: $179 \pm 23 \times 10^{4}$ and $131 \pm 25 \times 10^{4}$, respectively. The uptake of the contrast 
Table 3 Spearman rank order correlations between EMM parameters and histological and biochemical parameters

\begin{tabular}{|c|c|c|c|c|c|c|c|c|c|}
\hline & $T_{\text {peak }}[\min ]$ & $A$ & $\alpha[1 / \mathrm{min}]$ & $\beta[1 / \mathrm{min}]$ & $\gamma[1 / \mathrm{min}]$ & $q$ & $T_{1 / 2}[\mathrm{~min}]$ & ES & AUC \\
\hline Haemorrhagic necrosis [\%] & 0.588 & 0.279 & -0.558 & -0.224 & 0.282 & -0.398 & 0.429 & -0.579 & 0.322 \\
\hline Coagulative necrosis [\%] & 0.880 & 0.059 & -0.522 & -0.665 & 0.215 & -0.445 & 0.748 & -0.782 & 0.635 \\
\hline Vessel wall infiltration [a.u.] & 0.789 & 0.111 & -0.410 & -0.723 & 0.401 & -0.429 & 0.756 & -0.510 & 0.841 \\
\hline Tissue changes [\%] & 0.907 & 0.000 & -0.482 & -0.707 & 0.229 & -0.509 & 0.853 & -0.810 & 0.721 \\
\hline ALT [U/l] & 0.828 & -0.159 & -0.441 & -0.700 & 0.216 & -0.654 & 0.784 & -0.733 & 0.815 \\
\hline AST [U/l] & 0.776 & -0.095 & -0.332 & -0.723 & 0.090 & -0.574 & 0.789 & -0.631 & 0.859 \\
\hline
\end{tabular}

Correlations were calculated for seven animals in the control group and seven in the ALF group. Correlations marked in italics are assumed significant at $p<0.05$

agent from blood by hepatocytes was also disturbed in the ConA group as confirmed by the $T_{\text {peak }}$ and ES parameters. The maximum enhancement of image intensity was noticed at $6.14 \pm 1.07 \mathrm{~min}$ in the control group, while in the ConA group it occurred later at $9.72 \pm 1.69 \mathrm{~min}$. The ES value of $6587 \pm 1731$ and $4002 \pm 11131 / \mathrm{min}$ in the control and the ConA group, respectively, confirmed that perfusion in the ConA animals was affected by ALF.

\section{Correlations between MRI-based parameters and histopathological and biochemical outcomes}

The Spearman rank order correlation showed that perfusion measured with ASL correlates with the parameters calculated with EMM: $\alpha\left(r_{\mathrm{S}}=0.635\right), \gamma\left(r_{\mathrm{S}}=-0.591\right)$, $T_{\text {peak }}\left(r_{\mathrm{S}}=-0.732\right)$ and $T_{1 / 2}\left(r_{\mathrm{S}}=-0.609\right)$. Perfusion also correlates with $T_{1}\left(r_{\mathrm{S}}=-0.534\right)$ and two histopathological outcomes: vessel wall infiltration $\left(r_{\mathrm{S}}=-0.766\right)$ and tissue changes $\left(r_{\mathrm{S}}=-0.565\right)$. The $T_{1}$ relaxation time correlated with perfusion (in the text), $T_{\text {peak }}\left(r_{\mathrm{S}}=0.754\right), \beta$ $\left(r_{\mathrm{S}}=-0.640\right), T_{1 / 2}\left(r_{\mathrm{S}}=0.859\right), \mathrm{ES}\left(r_{\mathrm{S}}=-0.582\right)$ and $\operatorname{AUC}\left(r_{\mathrm{S}}=0.613\right)$, coagulative necrosis $\left(r_{\mathrm{S}}=0.689\right)$, vessel wall infiltration $\left(r_{\mathrm{S}}=0.785\right)$, tissue changes $\left(r_{\mathrm{S}}=0.801\right)$, $\operatorname{ALT}\left(r_{\mathrm{S}}=0.716\right)$ and AST $\left(r_{\mathrm{S}}=0.736\right)$. Correlations between EMM and histological as well as biochemical parameters were also found (Table 3). All histological parameters were positively correlated with the biochemical markers $(p<0.05)$.

\section{Discussion}

The aim of this study was to measure perfusion and hepatocyte injury in a mouse model of ALF induced with ConA using two MRI-based methods. The results demonstrated that the DCE-MRI and ASL techniques allow reliable assessment of alterations in liver perfusion and hepatocyte integrity induced by ConA in vivo.

ConA-induced liver injury is an established model of T-cell-mediated liver inflammation that to some extent mirrors autoimmune or viral hepatitis in humans [10]. The activated lymphocytes cause the release of pro-inflammatory mediators (e.g. INF $\gamma, \mathrm{TNF} \alpha, \mathrm{IL}-4)$, disruption of the endothelium and hepatocyte damage [10, 26, 27]. Our study, performed $24 \mathrm{~h}$ after ConA administration, allowed observing coagulative and haemorrhagic necrosis, inflammatory infiltration in the blood vessel walls and liver parenchyma as well as elevated levels of biochemical markers. All changes resembled the lesions in hepatitis, confirming the previous reports on this model $[6,28]$.

The ASL study showed that perfusion in the liver parenchyma in the control animals was $245 \pm 20 \mathrm{ml} / \mathrm{min} / 100 \mathrm{~g}$, which is in good agreement with the previously reported values of $220 \pm 30 \mathrm{ml} / \mathrm{min} / 100 \mathrm{~g}$ in Balb/c mice obtained using the FAIR-ASL technique with the Look-Locker readout [29]. In our study, perfusion in ALF was impaired by $18 \%$, likely due to the inflow of activated and therefore inflexible and more viscous leucocytes into the site of inflammation [30-32]. The sinusoids may also be plugged by the active platelets, which circulate in blood and are not cleared by the damaged liver [8, 9]. However, some authors indicated that ALF was associated with inappropriate vasodilation and vasoconstriction [7, 9].

The $T_{1}$ relaxation time was higher in the ConA group than in the control group. The $T_{1}$ value in the control group was $1249 \pm 91 \mathrm{~ms}$, which is comparable to the previously reported $1360 \pm 60 \mathrm{~ms}$ [29]. The correlation between $T_{1}$ and perfusion suggested that the change observed in the value of the relaxation time is linked with the haemodynamics. The increased $T_{1}$ time in the ConA group may be caused by water accumulation in the diseased tissue. Blood flow through the liver may be inefficient because of vessel embolisms with platelets and sequestrate leucocytes as well the reduction of the sinusoid size caused by the disturbed vasoconstrictors and vasodilator secretion [9].

Although liver function assessed by the DCE technique is also determined by liver perfusion, hepatic uptake of the Gd-EOB-DTPA contrast agent is strongly dependent on the hepatocyte integrity as Gd-EOB-DTPA is selectively taken up by the hepatocytes via the organ-anion transporters 
(OATPB1/B3) and excreted via ATP-dependent multidrug resistance proteins (MRP2/3/4) [24, 33, 34]. The high values of the $\mathrm{R}^{2}$ coefficients confirmed a very good fit of the EMM equation [25] to the DCE-MRI data. No differences between the animal groups in the rate of contrast uptake $(\alpha)$, initial rate of contrast uptake $(q)$ and maximal amplitude of image enhancement $(A)$ were observed. Interestingly, $\alpha$ correlated with the perfusion value obtained using ASL. Furthermore a difference was observed in the $T_{\text {peak }}$ and ES parameters, which may indicate alterations in the blood circulation, liver filtering function and hepatocyte integrity. The $\alpha, T_{\text {peak }}$ and ES parameters were previously assigned as perfusion indicators $[19,20]$, which was confirmed in our study by the obtained correlations. At the same time we also observed correlations of these parameters with pathological changes in histological staining, supporting the theory that contrast uptake and signal enhancement depend on several factors. The wash-out slope of the DCE-MRI curve in the ConA animals was affected by disturbed transport of the contrast agent via hepatocytes because of inflammation and the recovery processes. The differences between the groups were visible in the shape of the wash-out slope and the intensities of the liver images obtained during the final stage of MR experiments. The deteriorated liver function was apparent in the elimination half-life time $\left(T_{1 / 2}\right)$ value and the rate of the contrast washout $(\beta)$. Both of these parameters indicated significantly prolonged contrast removal in the ConA group. Their good correlation with histology (coagulative necrosis, tissue changes and vessel wall infiltrations) as well as blood markers implies an association with damaged hepatocytes. Although the differences observed in the histological samples between the groups are larger when compared to the differences obtained in the MRI parameters, it should be stressed that MRI provides in vivo measurements corresponding to pathophysiological processes and enables noninvasively assessing the progression or regression of the liver disease.

The drawback of the ASL technique is its low in-plane spatial resolution $(0.469 \mathrm{~mm} / \mathrm{pixel})$. The low resolution of the obtained images and need to remove the pixels with extreme values introduced additional complications to the data processing and analysis. The $500 \mathrm{ml} / \mathrm{min} / 100 \mathrm{~g}$ threshold was applied to exclude the blood vessels and the pixels with negative values from the MR images. Additionally, the circulatory bed in the liver has a complex structure containing not only arteries and veins but also the portal triads and sinusoid. Hence it is difficult to extract only the parenchymal area in the image.

DCE-MRI data were collected using the self-triggered IntraGateFLASH ${ }^{\mathrm{TM}}$ sequence. In this method cardiac and respiration cycles are derived from the MR signal and are used to reduce artifacts without sacrificing temporal resolution, which is especially important during the first passage of the bolus, providing information on perfusion $[35,36]$. The data in this study were collected at high temporal resolution (less than $4 \mathrm{~s}$ per image) during the contrast wash-in phase.

Clinical evaluation of patients with ALF is based on the King's College Hospital criteria, which incorporate the aetiology of the disease and blood tests [37]. The imaging modalities used in the diagnosis of ALF include X-ray imaging, Doppler ultrasound, electroencephalography (ECG) and echocardiograms [37]. Nevertheless, MR has been used in clinical studies of hepatitis, mostly for the assessment of liver fibrosis and cirrhosis in chronic liver failure using MR elastography [38, 39], diffusion-weighted MRI [40, 41] and DCE-MRI [41-43].

The American College of Radiologists [44] indicated that MRI, with or without a contrast agent, is a suitable technique for clinical imaging of several liver diseases. The application of ASL to measure liver perfusion in humans has been also reported with promising results [45, 46]. Hence the results of our research, supported by the previous studies, suggest possible applications of our approach to clinics, in particular for assessing ALF physiology and patient monitoring, either during onset of the liver disease or, for example, after liver transplantation.

\section{Conclusion}

We have demonstrated that retrospectively gated dynamic contrast-enhanced MRI (DCE-MRI) with the hepatocyte-specific contrast agent and arterial spin labelling (ASL) technique allows monitoring of the alterations in hepatocyte integrity and in perfusion of the microcirculation in murine hepatitis induced by ConA. The results correlated with the histopathological and biochemical parameters of liver inflammation and injury. The results suggest that both the self-triggered IntraGateFLASH $^{\mathrm{TM}}$ and FAIR-EPI sequences are well suited for comprehensive imaging of liver injury in mice at 9.4 T. This method could be used to investigate the effects of pharmacological treatment on the progression or regression of liver disease in other models of liver injury. The IntraGateFLASH ${ }^{\mathrm{TM}}$ and FAIR-EPI pulse sequences could be implemented in the clinical settings to potentially improve the ALF diagnosis in patients by simultaneous assessment of alterations in liver perfusion and hepatocyte integrity in hepatitis or other liver injuries. Hence the method may prove useful in the diagnosis and therapy of liver diseases.

Acknowledgments This work was supported by the European Union for European Regional Development Fund (grant coordinated by the 
Jagiellonian Centre for Experimental Therapeutics-Jagiellonian University, No. WND-POIG.01.01.02-00-069/09-00) and partially by National Science Centre Grant No. DEC-2015/16/W/NZ4/00070.

\section{Compliance with ethical standards}

Conflict of interest The authors declare that they have no conflict of interest.

Welfare of animals All applicable national and institutional guidelines for the care and use of animals were followed. All procedures were approved by the local Ethics Committee on Animal Testing.

Open Access This article is distributed under the terms of the Creative Commons Attribution 4.0 International License (http://creativecommons.org/licenses/by/4.0/), which permits unrestricted use, distribution, and reproduction in any medium, provided you give appropriate credit to the original author(s) and the source, provide a link to the Creative Commons license, and indicate if changes were made.

\section{References}

1. Bernal W, Wendon J (2013) Acute liver failure. N Engl J Med 369(26):2525-2534

2. Sass DA, Shakil AO (2005) Fulminant hepatic failure. Liver Transpl 11(6):594-605

3. O'Grady JG (2005) Acute liver failure. Postgrad Med J 81(953):148-154

4. Berry P, Antoniades C, Hussain M, McPhail M, Bernal W, Vergani D, Wendon J (2010) Admission levels and early changes in serum interleukin-10 are predictive of poor outcome in acute liver failure and decompensated cirrhosis. Liver Int 30(5):733-740

5. Antoniades CG, Berry PA, Wendon JA, Vergani D (2008) The importance of immune dysfunction in determining outcome in acute liver failure. J Hepatol 49(5):845-861

6. Sass G, Heinlein S, Agli A, Bang R, Schümann J, Tiegs G (2002) Cytokine expression in three mouse models of experimental hepatitis. Cytokine 19(3):115-120

7. Trewby P, Williams R (1977) Pathophysiology of hypotension in patients with fulminant hepatic failure. Gut 18(12):1021-1026

8. Hughes R, Wendon J, Gimson A (1991) Acute liver failure. Gut 32(Suppl):S86-S91

9. Vollmar B, Menger MD (2009) The hepatic microcirculation: mechanistic contributions and therapeutic targets in liver injury and repair. Physiol Rev 89(4):1269-1339

10. Tiegs G, Hentschel J, Wendel A (1992) AT cell-dependent experimental liver injury in mice inducible by concanavalin A. J Clin Invest 90(1):196

11. Gantner F, Leist M, Lohse AW, Germann PG, Tiegs G (1995) Concanavalin A-induced T-cell-mediated hepatic injury in mice: the role of tumor necrosis factor. Hepatology 21(1):190-198

12. Knolle PA, Gerken G, Löser E, Dienes H-P, Gantner F, Tiegs G, Meyer zum Büschenfelde K, Lohse AW (1996) Role of sinusoidal endothelial cells of the liver in concanavalin A-induced hepatic injury in mice. Hepatology 24(4):824-829

13. Detre JA, Leigh JS, Williams DS, Koretsky AP (1992) Perfusion imaging. Magn Reson Med 23(1):37-45

14. Zhou XJ (2004) Arterial Spin Tagging. In: Bernstein MA, King KF, Zhou XJ (eds) Handbook of MRI pulse sequences, vol 1. Elsevier Academic Press, USA, pp 802-829
15. Chow AM, Gao DS, Fan SJ, Qiao Z, Lee FY, Yang J, Man K, Wu EX (2012) Measurement of liver T1 and T2 relaxation times in an experimental mouse model of liver fibrosis. J Magn Reson Imaging 36(1):152-158

16. Stark D, Felder R, Wittenberg J, Saini S, Butch R, White M, Edelman R, Mueller P, Simeone J, Cohen A (1985) Magnetic resonance imaging of cavernous hemangioma of the liver: tissue-specific characterization. AJR Am J Roentgenol 145(2):213-222

17. Moriyama Y, Saito S, Kobayashi S, Ogihara R, Koto D, Kitamura A, Matsushita T, Nishiura M, Murase K (2012) Evaluation of concanavalin A-induced acute liver injury in rats using an empirical mathematical model and dynamic contrast-enhanced MR imaging with Gd-EOB-DTPA. Magn Reson Med Sci 11(1):53-60

18. Goshima S, Kanematsu M, Watanabe H, Kondo H, Kawada $H$ Moriyama N, Bae KT (2012) Gd-EOB-DTPA-enhanced MR imaging: prediction of hepatic fibrosis stages using liver contrast enhancement index and liver-to-spleen volumetric ratio. J Magn Reson Imaging 36(5):1148-1153

19. Positano V, Santarelli MF, Landini L (2003) Automatic characterization of myocardial perfusion in contrast enhanced MRI. EURASIP J Appl Signal Process 2003:413-421

20. Tarroni G, Corsi C, Antkowiak PF, Veronesi F, Kramer CM, Epstein FH, Walter J, Lamberti C, Lang RM, Mor-Avi V (2012) Myocardial perfusion: near-automated evaluation from contrastenhanced MR images obtained at rest and during vasodilator stress. Radiology 265(2):576-583

21. Bovens SM, te Boekhorst B, Ouden Kd, van de Kolk KW, Nauerth A, Nederhoff MG, Pasterkamp G, ten Hove M, van Echteld CJ (2011) Evaluation of infarcted murine heart function: comparison of prospectively triggered with self-gated MRI. NMR Biomed 24(3):307-315

22. Coolen BF, Geelen T, Paulis LE, Nauerth A, Nicolay K, Strijkers GJ (2011) Three-dimensional T1 mapping of the mouse heart using variable flip angle steady-state MR imaging. NMR Biomed 24(2):154-162

23. Tabibian JH, Macura SI, O'Hara SP, Fidler JL, Glockner JF, Takahashi N, Lowe VJ, Kemp BJ, Mishra PK, Tietz PS (2013) Micro-computed tomography and nuclear magnetic resonance imaging for noninvasive, live-mouse cholangiography. Lab Invest 93(6): 733-743

24. Van Beers BE, Pastor CM, Hussain HK (2012) Primovist, Eovist: what to expect? J Hepatol 57(2):421-429

25. Fan X, Medved M, Karczmar GS, Yang C, Foxley S, Arkani S, Recant W, Zamora MA, Abe H, Newstead GM (2007) Diagnosis of suspicious breast lesions using an empirical mathematical model for dynamic contrast-enhanced MRI. Magn Reson Imaging 25(5):593-603

26. Sternak M, Jakubowski A, Czarnowska E, Slominska EM, Smolenski RT, Szafarz M, Walczak M, Sitek B, Wojcik T, Jasztal A (2015) Differential involvement of IL-6 in the early and late phase of 1-methylnicotinamide (MNA) release in Concanavalin A-induced hepatitis. Int Immunopharmac 28(1):105-114

27. Mizuhara H, O’Neill E, Seki N, Ogawa T, Kusunoki C, Otsuka K, Satoh S, Niwa M, Senoh H, Fujiwara H (1994) T cell activation-associated hepatic injury: mediation by tumor necrosis factors and protection by interleukin 6. J Exp Med 179(5):1529-1537

28. Sternak M, Khomich TI, Jakubowski A, Szafarz M, Szczepański W, Białas M, Stojak M, Szymura-Oleksiak J, Chłopicki S (2010) Nicotinamide $N$-methyltransferase (NNMT) and 1-methylnicotinamide (MNA) in experimental hepatitis induced by concanavalin A in the mouse. Pharm Rep 62(3):483-493

29. Ramasawmy R, Campbell-Washburn A, Wells J, Johnson S, Pedley R, Walker-Samuel S, Lythgoe M (2015) Hepatic arterial 
spin labelling MRI: an initial evaluation in mice. NMR Biomed 28(2):272-280

30. Bauer M, Marzi I, Thuma B, Bach F, Bühren V, Larsen R (1994) Dose-related pattern of sinusoidal leukocyte adhesion in sublobular regions of the liver after systemic endotoxin challenge in the rat. Shock 1(2): 135-140

31. Frank RS (1990) Time-dependent alterations in the deformability of human neutrophils in response to chemotactic activation. Blood 76(12):2606-2612

32. Downey GP, Elson EL, Schwab B, Erzurum SC, Young SK, Worthen GS (1991) Biophysical properties and microfilament assembly in neutrophils: modulation by cyclic AMP. J Cell Biol 114(6): 1179-1190

33. Verloh N, Utpatel K, Haimerl M, Zeman F, Fellner C, FichtnerFeigl S, Teufel A, Stroszczynski C, Evert M, Wiggermann P (2015) Liver fibrosis and Gd-EOB-DTPA-enhanced MRI: a histopathologic correlation. Sci Rep 5:15408

34. Nojiri S, Kusakabe A, Fujiwara K, Shinkai N, Matsuura K, Iio E, Miyaki T, Joh T (2013) Noninvasive evaluation of hepatic fibrosis in hepatitis $\mathrm{C}$ virus-infected patients using ethoxybenzyl-magnetic resonance imaging. J Gastroenterol Hepatol 28(6):1032-1039

35. Sourbron S (2010) Technical aspects of MR perfusion. Eur $\mathbf{J}$ Radiol 76(3):304-313

36. Khalifa F, Soliman A, El-Baz A, El-Ghar MA, El-Diasty T, Gimel'farb G, Ouseph R, Dwyer AC (2014) Models and methods for analyzing DCE-MRI: a review. Med Phys 41(12):124301

37. Pathikonda M, Munoz SJ (2010) Acute liver failure. Ann Hepatol 9(1):7-14

38. Huwart L, Peeters F, Sinkus R, Annet L, Salameh N, ter Beek LC, Horsmans Y, Van Beers BE (2006) Liver fibrosis: noninvasive assessment with MR elastography. NMR Biomed 19(2):173-179

39. Rouviere O, Yin M, Dresner MA, Rossman PJ, Burgart LJ, Fidler JL, Ehman RL (2006) MR elastography of the liver: preliminary results 1 . Radiology 240(2):440-448
40. Taouli B, Chouli M, Martin AJ, Qayyum A, Coakley FV, Vilgrain V (2008) Chronic hepatitis: role of diffusion-weighted imaging and diffusion tensor imaging for the diagnosis of liver fibrosis and inflammation. J Magn Reson Imaging 28(1):89-95

41. Patel J, Sigmund EE, Rusinek H, Oei M, Babb JS, Taouli B (2010) Diagnosis of cirrhosis with intravoxel incoherent motion diffusion MRI and dynamic contrast-enhanced MRI alone and in combination: preliminary experience. J Magn Reson Imaging 31(3):589-600

42. Chen B-B, Hsu C-Y, Yu C-W, Wei S-Y, Kao J-H, Lee H-S, Shih TT-F (2012) Dynamic contrast-enhanced magnetic resonance imaging with Gd-EOB-DTPA for the evaluation of liver fibrosis in chronic hepatitis patients. Eur Radiol 22(1):171-180

43. Norén B, Forsgren MF, Leinhard OD, Dahlström N, Kihlberg J, Romu T, Kechagias S, Almer S, Smedby Ö, Lundberg P (2013) Separation of advanced from mild hepatic fibrosis by quantification of the hepatobiliary uptake of Gd-EOB-DTPA. Eur Radiol 23(1):174-181

44. Nelson RC, Kamel I, Baker M, Al-Refaie W, Cash B, Harrison S, Hindman N, Kaur H, McNamara M, Qayyum A, Tulchinsky M, Yarmish G, Rosen M (2014) ACR Appropriateness criteria ${ }^{\circledR}$ liver lesion-initial characterization. http://www.guideline.gov/ content.aspx?id=48283. Accessed 7 April 2016

45. Katada Y, Shukuya T, Kawashima M, Nozaki M, Imai H, Natori T, Tamano M (2012) A comparative study between arterial spin labeling and CT perfusion methods on hepatic portal venous flow. Jpn J Radiol 30(10):863-869

46. Cox E, Ghezzi A, Patel M, Jackson A, Costigan C, James M, Ryder S, Gowland P, Aithal G, Francis S (2012) A novel MRI protocol to examine haemodynamic compartments in compensated liver cirrhosis. Gut 61(Suppl 2):A195-A196 